\title{
Numerical simulation of the viscous interaction of the solar wind with the magnetic polar regions of Venus
}

\author{
M. Reyes-Ruiz ${ }^{1}$, E. Díaz-Méndez ${ }^{1}$, and H. Pérez-de-Tejada ${ }^{2}$ \\ ${ }^{1}$ Instituto de Astronomía, Universidad Nacional Autónoma de México, Apdo Postal 877, Ensenada 22800, B.C., México \\ e-mail: [maurey; ediaz] @astrosen.unam.mx \\ 2 Instituto de Geofísica, Universidad Nacional Autónoma de México, Ciudad Universitaria, C.P. 04510, D.F., México \\ e-mail: perezdet@geofisica.unam.mx
}

Received 17 December 2007 / Accepted 5 June 2008

\begin{abstract}
Aims. We aim to study the viscous interaction of the solar wind with the ionosphere of Venus in the magnetic polar regions by means of hydrodynamical computer simulations.

Methods. We use a finite difference code developed for the solution of the Navier-Stokes, continuity and energy equations.

Results. We have calculated the flow properties, the shape and location of the boundary layer and the shock formed as a consequence of the viscous interaction over the magnetic poles of the planet and advected downstream. By comparing our results to the flow properties measured by the Venera 10 and Mariner 5 spacecraft in the Venus ionosheath, we determine that a Reynolds number for the flow near the value $R e=20$ is necessary to reproduce the position of the intermediate transition as well as the shock front. Also, on the basis of our results we predict the existence of a stagnation region for the solar wind flow extending considerably upstream from the viscous interaction region.
\end{abstract}

Key words. planets and satellites: individual: Venus - interplanetary medium - solar wind - methods: numerical

\section{Introduction}

In situ measurements of the physical environment in the ionosphere and ionosheath over the magnetic polar regions of the planet Venus indicate that many observed features can be best understood as arising from the viscous interaction of the solar wind plasma with ionospheric plasma over the polar regions of the planet (see Pérez-de-Tejada 1995, 2006 and references therein). We briefly review the main features believed to be related to viscous transport processes in the ionosheath of Venus.

Both the Mariner 5 and Venera 10 spacecraft observed a reduction of the flow velocity as they transversed the ionosheath moving towards the planet (Romanov et al. 1979; Bridge et al. 1967). The accompanying decrease of plasma density and the increase in the gas temperature as the spacecraft approached the polar regions of the planet are consistent with the existence of a viscous boundary layer arising from the direct plasma-plasma interaction at the ionopause over the magnetic polar regions of the planet (Pérez-de-Tejada et al. 1995).

According to Pérez-de-Tejada (1986a) the transterminator flow observed by the Pioneer Venus Orbiter (PVO), i.e. the dayside to nightside flow of upper ionospheric plasma with typical speeds of a few $\mathrm{km} \mathrm{s}^{-1}$, is consistent with the viscous dragging of the Venus upper ionosphere by the solar wind flow in the ionosheath above the magnetic polar regions. Pérez-de-Tejada (1986a) has shown that the altitude integrated momentum flux in the transterminator flow is comparable to the deficiency of solar wind momentum as measured by Mariner 5 in the ionosheath over the magnetic poles of Venus (Bridge et al. 1967; Shefer et al. 1979).

It has been argued that the direct, viscous interaction between the solar wind plasma and ionospheric plasma occurs preferentially over the polar regions due to the shielding provided by the piled-up IMF elsewhere over the planet's ionosphere (Pérez-de-Tejada 1986b). Pérez-de-Tejada (2001, 2004) has proposed that the viscous interaction leads to the carving out of plasma channels at and downstream of the magnetic polar regions providing an explanation for the ionospheric holes measured by the PVO on the nightside of the planet (Brace et al. 1983; Taylor et al. 1985).

The precise mechanisms responsible for the momentum transport processes we describe in terms of an anomalous viscosity have been addressed by Shapiro et al. (1995) and Quest et al. (1997). They propose that viscosity could be related to wave-particle interactions given the strongly fluctuating magnetic field in the ionosheath as measured by Mariner 5 . However, it is clear that the precise origin of the anomalous viscosity is not fully understood at present and is beyond the scope of the present paper.

In this paper we present the basic properties of a numerical code developed to simulate the viscous interaction of the solar wind with the planet Venus. As part of this ongoing effort, in this paper we report a first attempt to gain insight into this problem by concentrating on the flow properties above the magnetic poles of the planet. The paper is organized as follows; in Sect. 2 we describe the basic equations and assumptions of the model used to study the viscous interaction, in Sect. 3 we briefly describe the numerical code we are using and other numerical details. Results for a series of cases illustrating the effect of the various model parameters are shown in Sect. 4. In Sect. 5 we discuss these results in the context of in situ measurements made by the Venera 10 and Mariner 5 spacecraft. Finally, in Sect. 6 we summarize our findings and discuss our results. 


\section{Problem setup}

We assume that a direct, viscous interaction between the solar wind plasma and the ionospheric plasma of the planet Venus occurs only at very high latitudes. Whitten et al. (1984) has estimated that the region of plasma-plasma interaction extends only from about $80^{\circ}$ solar zenith angle (SZA) to approximately $140^{\circ}$ SZA over each magnetic pole (as traditionally defined in terms of the prevailing IMF). This suggests that, as a first approximation, one may neglect curvature effects entirely and consider the boundary of the ionosphere where the direct interaction occurs as a flat plate. Also, as extensively measured by PVO and other spacecraft (Miller \& Whitten 1991), flow velocities in the upper ionosphere are generally lower than $5 \mathrm{~km} \mathrm{~s}^{-1}$, much smaller than the speed of the free flowing solar wind as it streams over the planet's polar regions. This suggests that a no-slip boundary condition, with the flat plate taken at rest, is a good approximation for the scenario we want to model.

We consider only fluid forces in our model, i.e. we do not include the effect of the IMF, mass loading or any body forces. An estimation of the effect of $\boldsymbol{J} \times \boldsymbol{B}$ forces in comparison to viscous forces in the ionosheath has been carried out by Pérez-de-Tejada (1999). Briefly, an order of magnitude comparison of the advection, magnetic and viscous terms in the $x$-component of the momentum equation in the boundary layer yields:

$\frac{\rho_{\mathrm{o}} U_{\mathrm{o}}^{2}}{L} \sim \frac{B_{\mathrm{o}}^{2}}{4 \pi L}+\rho_{\mathrm{o}} \frac{v U_{\mathrm{o}}}{\delta^{2}}$

where $\rho_{\mathrm{o}}, U_{\mathrm{o}}$ and $B_{\mathrm{o}}$ are typical magnitudes of the density, flow velocity and magnetic field respectively, $v$ is kinematic viscosity, $L$ is the characteristic length scale in the $x$ direction and $\delta$ is the thickness of the boundary layer. Using density and velocity values for the solar wind in the ionosheath this equation can be recast as:

$\rho_{\mathrm{SW}} U_{\mathrm{SW}}^{2} \sim \rho_{\mathrm{SW}} U_{\mathrm{SW}}^{2}\left[\frac{V_{\mathrm{A}}^{2}}{2 U_{\mathrm{SW}}^{2}}+\frac{1}{R e} \frac{L^{2}}{\delta^{2}}\right]$

where $V_{\mathrm{A}}$ is the Alfven velocity and $R e$ is the Reynolds number of the flow. Using $\delta \sim L / \sqrt{R e}$ as in a Blasius boundary layer we see that the relative importance of both terms is given by:

$\frac{\boldsymbol{J} \times \boldsymbol{B} \text { forces }}{\text { viscous forces }} \sim \frac{1}{2 M_{\mathrm{A}}^{2}}$.

As discussed by Pérez-de-Tejada (1999) the flow over the polar regions being super-Alfvenic implies that magnetic forces are not the dominant factor when studying flow dynamics in the region.

To estimate the importance of mass loading consider that the total amount of momentum taken from the solar wind flow in the ionosheath is, at most, the amount of momentum contained in the flow of $\mathrm{O}^{+}$ions of ionospheric origin in the region, as they start essentially from rest. The dynamical importance of this process can be estimated by comparing the kinetic energy in the flow of $\mathrm{O}^{+}$ions, $\rho_{\mathrm{O}^{+}} U_{\mathrm{O}^{+}}^{2} / 2$, to the kinetic energy in the solar wind proton flow, $\rho_{\mathrm{SW}} U_{\mathrm{SW}}^{2} / 2$. Since the velocity of the $\mathrm{O}^{+}$ions, $U_{\mathrm{O}^{+}}$is, at most, equal to the velocity of the solar wind protons, $U_{\mathrm{SW}}$, an upper limit on the relative importance of mass loading on the flow dynamics is given by the comparison of $\rho_{\mathrm{O}^{+}}$and $\rho_{\mathrm{SW}}$. According to data from August 11, 2006 from the ASPERA4 instrument on board the Venus Express spacecraft (Lundin, private communication), the mass density of $\mathrm{O}^{+}$ions in the ionosheath, and consequently also the kinetic energy density, is approximately an order of magnitude lower than that for $\mathrm{H}^{+}$.

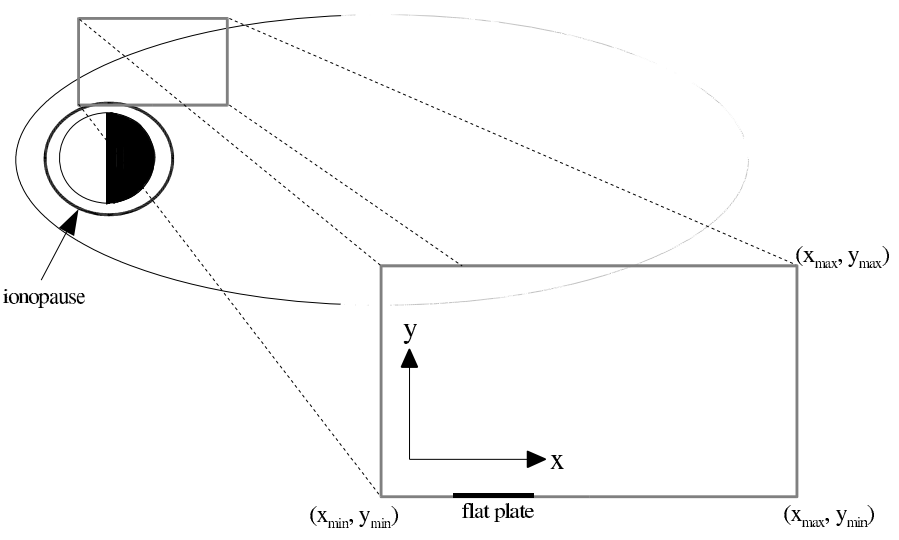

Fig. 1. Schematics of the geometry of our problem. The gray box represents the region we are modeling. The Sun is to the left and the flat plate represents that portion of the ionopause where the direct, viscous interactions between the solar wind and ionospheric plasma takes place. Not to scale.

At present, we consider only the problem in 2D, $x$ and $y$, where $x$ represents the distance along the plate, i.e. along the Sun-Venus direction in our model, and $y$ represents the height above the flat plate, which represents the ionopause in our model.

In summary, we propose to model the viscous interaction of the solar wind with the ionosphere at the magnetic polar regions of the planet Venus as a hydrodynamic, 2D, initially uniform flow along a finite flat plate. Figure 1 illustrates the geometry of our problem. In terms of our model, the flat plate represents the portion of Venus ionopause over which there is a direct interaction of solar wind and ionospheric plasma.

The full Navier-Stokes equations are solved together with the continuity and energy equations in conservative form which, after passing to dimensionless variables, can be written as:

$\frac{\partial \boldsymbol{U}}{\partial t}=\frac{\partial \boldsymbol{E}}{\partial x}+\frac{\partial \boldsymbol{F}}{\partial y}$

where

$\boldsymbol{U}=\left(\begin{array}{c}\rho \\ \rho V_{x} \\ \rho V_{y} \\ E_{\mathrm{t}}\end{array}\right)$,

$\boldsymbol{E}=\left(\begin{array}{c}\rho V_{x} \\ \rho V_{x} V_{x}+k_{1} p-k_{2} T_{x x} \\ \rho V_{x} V_{y}-k_{2} T_{x y} \\ \left(E_{\mathrm{t}}+k_{3} p\right) V_{x}-k_{4}\left(V_{x} T_{x x}+V_{y} T_{x y}\right)+k_{5} \dot{q}_{x}\end{array}\right)$

and

$$
\boldsymbol{F}=\left(\begin{array}{c}
\rho V_{y} \\
\rho V_{x} V_{y}-k_{2} T_{x y} \\
\rho V_{y} V_{y}+k_{1} p-k_{2} T_{y y} \\
\left(E_{\mathrm{t}}+k_{3} p\right) V_{y}-k_{4}\left(V_{x} T_{x y}+V_{y} T_{y y}\right)+k_{5} \dot{q}_{y}
\end{array}\right) .
$$


In the preceding equations $\rho$ is the gas density, $V_{x}$ and $V_{y}$ are the components of the velocity and $E_{\mathrm{t}}$ is the total energy density defined by;

$E_{\mathrm{t}}=\rho e+\frac{1}{2} \rho V^{2}$

with $e$ being the internal energy per unit mass. In (3) and (4), $k_{i}$ for $i=1,5$ contain the following combinations of dimensionless numbers and the adiabatic index for the gas, $\gamma$ :

$k_{1}=\frac{1}{\gamma M_{\mathrm{o}}^{2}}$

$k_{2}=\frac{1}{R e}$

$k_{3}=(\gamma-1)$

$k_{4}=\frac{\gamma(\gamma-1) M_{\mathrm{o}}^{2}}{\operatorname{Re}}$

$k_{5}=\frac{\gamma}{\operatorname{RePr}}$.

The Mach, Reynolds and Prandtl numbers for the flow, $M_{\mathrm{o}}, R e$, and $\operatorname{Pr}$ respectively, are defined as:

$M_{\mathrm{o}}=\frac{V_{\mathrm{o}}}{C_{s o}}$

$\operatorname{Re}=\frac{V_{\mathrm{o}} L}{\rho_{\mathrm{o}} \mu_{\mathrm{o}}}$

$\operatorname{Pr}=\frac{\mu_{\mathrm{o}} c_{\mathrm{p}}}{\kappa_{\mathrm{o}}}$

where quantities with subindex o are those used for the normalization of the flow variables and parameters, $c_{\mathrm{p}}$ is the specific heat at constant pressure and $L$ is the normalization for the spatial coordinates. For simplicity, and in the absence of arguments to do otherwise, we have assumed that flow parameters $\mu$ and $\kappa$ are uniform and equal to $\mu_{\mathrm{o}}$ and $\kappa_{\mathrm{o}}$.

$T_{x x}, T_{x y}$ and $T_{y y}$ in Eqs. (3) and (4) are components of the viscous stress tensor given by:

$T_{x x}=\frac{4}{3} \frac{\partial V_{x}}{\partial x}-\frac{2}{3} \frac{\partial V_{y}}{\partial y}$

$T_{x y}=\frac{\partial V_{y}}{\partial x}+\frac{\partial V_{x}}{\partial y}$

and

$T_{y y}=-\frac{2}{3} \frac{\partial V_{x}}{\partial x}+\frac{4}{3} \frac{\partial V_{y}}{\partial y}$.

Also, in Eqs. (3) and (4), $\dot{q}_{x}$ and $\dot{q}_{y}$ are the components of the heat flux vector given by:

$\dot{q}_{x}=-\frac{\partial T}{\partial x}$

and

$\dot{q}_{y}=-\frac{\partial T}{\partial y}$.
Finally, we have assumed throughout that the gas is ideal so that:

$e=\frac{1}{\gamma-1} \frac{p}{\rho}$

with the ideal gas equation of state, $p=\rho R T$. For the calculations shown here we have assumed the gas to be monoatomic, i.e. $\gamma=5 / 3$.

In applying these set of equations to our flat plate model for the ionopause of Venus at polar latitudes, Eq. (1) is solved in a rectangular domain with the following boundary conditions:

- at $x=x_{\min }$ (left boundary) and at $y=y_{\max }$ (top boundary) the flow is assumed uniform and with the properties of the solar wind in the ionosheath at polar latitudes according to the non-viscous calculations of Spreiter \& Stahara (1980),

$V_{x}=V_{\mathrm{SW}}$,

$V_{y}=0$,

$p=p_{\mathrm{SW}}$,

$T=T_{\mathrm{SW}}$,

- At $x=x_{\max }$ (right boundary) the flow properties are assumed to continue evolving smoothly as it distances itself from the obstacle. All flow variables are linearly extrapolated in the $x$ direction.

- At $y=y_{\min }$ (bottom boundary) the boundary conditions depend on whether we are over the flat plate or not. The plate is taken to extend from $x_{\text {plate, } 1}$ to $x_{\text {plate, } 2 \text {. }}$.

For $x_{\text {plate }, 1}<x<x_{\text {plate }, 2}$ the following boundary conditions are used:

$V_{x}=0$,

$V_{y}=0$,

$\frac{\partial T}{\partial y}=0$,

and pressure, $p$, is extrapolated linearly from its values in the region immediately above the plate. The condition for a vanishing temperature gradient normal to the plate, implying no heat flow across the ionopause, is traditionally used in the solution of the flat plate, viscous problem and considered appropriate for the steady state solution we seek.

We choose our normalization constants such that $V_{\mathrm{SW}}=p_{\mathrm{SW}}=$ $T_{\mathrm{SW}}=1$, i.e. flow properties $V_{x}, V_{y}, \rho, p$ and $T$ are all normalized to their value in the solar wind as it enters the computational box. The length scale normalization, $L$, is chosen to be the size of the flat plate, which in our case represents the size of the ionopause region where the solar wind directly interacts with ionospheric plasma. If we consider that the interaction region extends from $80^{\circ}$ to $140^{\circ} \mathrm{SZA}$ over the magnetic poles of the planet, then $L \approx 6300 \mathrm{~km}$.

\section{The numerical code}

To address the problem described above, we have developed a computational code following Anderson (1995). The code solves Eq. (1) on a uniform, rectangular grid, using MacCormack's scheme which is 2 nd order accurate in space and time. Although we are solving the time-dependent equations for the flow, we seek only the steady state solution. This is reached by evolving the equations from a given set of initial conditions until there is no variation in the flow properties up to a user defined tolerance. In the cases shown this is typically achieved after fewer than 30000 iterations. 


\subsection{Finite difference scheme}

In MacCormack' scheme the solution is advanced over one timestep by a sequence of intermediate steps, the predictor and corrector steps. In the predictor step an intermediate solution is calculated from the values of the physical variables, $\boldsymbol{U}_{i, j}^{t}$ at a given time $(t)$ and position, $\left(x_{i}, y_{j}\right)$, according to:

$$
\begin{aligned}
\boldsymbol{U}_{i, j}^{*}=\boldsymbol{U}_{i, j}^{t} & -c_{1}\left[\boldsymbol{E}_{i+1, j}^{t}-\boldsymbol{E}_{i, j}^{t}\right] \\
& -c_{2}\left[\boldsymbol{F}_{i, j+1}^{t}-\boldsymbol{F}_{i, j}^{t}\right]
\end{aligned}
$$

where $c_{1}=\Delta t / \Delta x, c_{2}=\Delta t / \Delta y$ and $\boldsymbol{E}^{t}$ and $\boldsymbol{F}^{t}$ are evaluated with $\boldsymbol{U}^{t}$ according to (3) and (4). This predicted solution is then corrected to obtain the solution at the next time, $t+\Delta t$, using:

$$
\begin{aligned}
\boldsymbol{U}_{i, j}^{t+\Delta t}=\frac{1}{2}\left[\boldsymbol{U}_{i, j}^{t}\right. & +\boldsymbol{U}_{i, j}^{*}-c_{1}\left(\boldsymbol{E}_{i, j}^{*}-\boldsymbol{E}_{i-1, j}^{*}\right) \\
& \left.-c_{2}\left(\boldsymbol{F}_{i, j}^{*}-\boldsymbol{F}_{i, j-1}^{*}\right)\right]
\end{aligned}
$$

where $\boldsymbol{E}^{*}$ and $\boldsymbol{F}^{*}$ are computed from $\boldsymbol{U}^{*}$ using (3) and (4). Following Anderson (1995), in the evaluation of $\boldsymbol{E}^{t}$ we use first order backward differences for the $x$ derivatives and centered differences for the $y$ derivatives. For $\boldsymbol{F}^{t}$ we use centered differences for the $x$ derivatives and backward differences for the $y$ derivatives. For $\boldsymbol{E}^{*}$ we use forward differences for the $x$ derivatives and centered differences for the $y$ derivatives. For $\boldsymbol{F}^{*}$ we use centered differences for the $x$ derivatives and forward differences for the $y$ derivatives.

The time step, $\Delta t$, is calculated with the following version of the Courant-Friedrichs-Lewy criterion as suggested by Anderson (1995):

$\Delta t=\min \left[K\left(\Delta t_{\mathrm{CFL}}\right)_{i, j}\right]$

where $K$ is a constant, typically less than 0.5 , and

$$
\begin{aligned}
\left(\Delta t_{\mathrm{CFL}}\right)_{i, j}= & {\left[\frac{\left|\left(V_{x}\right)_{i, j}\right|}{\Delta x} \frac{\left|\left(V_{y}\right)_{i, j}\right|}{\Delta y}+\frac{1}{M_{\mathrm{o}}} \sqrt{\frac{1}{\Delta x^{2}}+\frac{1}{\Delta y^{2}}}\right.} \\
& \left.+\frac{2}{\operatorname{Re}}\left(\frac{1}{\Delta x^{2}}+\frac{1}{\Delta y^{2}}\right)\right]^{-1} .
\end{aligned}
$$

A full description of the code as well as the tests performed are reported in Díaz-Méndez (2007).

\subsection{Initial conditions}

The steady state solution for the flow is obtained by evolving the following initial conditions:

$$
\begin{aligned}
& V_{x}(t=0)=V_{\mathrm{SW}}, \\
& V_{y}(t=0)=0, \\
& p(t=0)=p_{\mathrm{SW}}, \\
& T(t=0)=T_{\mathrm{SW}} .
\end{aligned}
$$

Although significantly different from the final flow properties, these conditions do not give rise to any long lasting instability in the flow so that the final state does not depend on their precise form or value.

\section{Results}

Apart from the boundary conditions, the steady state solution for the flow depends on the value of 3 dimensionless numbers; the Mach number of the incident flow, $M_{\mathrm{o}}$, the Reynolds number of the flow, $R e$, and the Prandtl number, $P r$. In the following subsection we discuss what the fiducial values for $M_{\mathrm{o}}$ and $R e$ should be and present results for this case. Next, we present results for a series of cases with greater $M_{\mathrm{O}}$ and different $R e$ to illustrate the effect of each parameter. For the Prandtl number we use $\operatorname{Pr}=0.71$ in all our calculations. Such a value corresponds to a monoatomic ideal gas (Isachenko et al. 1977). Our results are not significantly different if we use a similar value, e.g. $\operatorname{Pr}=1$.

\subsection{Fiducial choice of parameters}

The appropriate value of $M_{\mathrm{o}}$ to use in our model is taken from the work of Spreiter \& Stahara (1980) who calculated the flow of the Solar Wind past Venus without taking into account viscous forces. As we have argued, we expect the viscous effects of the interaction between the solar wind and the ionospheric plasma to be important mainly around the magnetic polar regions of the planet. Hence, we consider the results of Spreiter \& Stahara (1980) as a good approximation for the flow at low latitudes, where viscosity is not expected to play a crucial role. According to Spreiter \& Stahara (1980) the shocked solar wind in the ionosheath, above the polar regions, is characterized by $M_{\mathrm{o}} \approx 2$.

The value of $R e$ is taken from the work of Pérez-deTejada (1999), who estimates the value of the Reynolds number based on the thickness of the boundary layer as seen by the Venera 10 and Mariner 5 spacecraft. Perez-de-Tejada (1986a) also estimated $R e$ by calculating the momentum transfer from the solar wind to the upper ionosphere as measured by PVO (Perez-de-Tejada 1986a) in the transterminator flow. We adopt $R e \approx 20$ as a fiducial value based on these results.

The flow for our basic model, characterized by $M_{\mathrm{o}}=2$ and $R e=20$, is shown in Fig. 2. For these calculations we have used a grid of $200 \times 400$ mesh points, extending from $x_{\min }=0$ to $x_{\max }=5$ and $y_{\min }=0$ to $y_{\max }=15$. Figure 2 shows only a portion of the computational box. In the choice of the vertical extent of the computational domain care must be taken so that both the shock front and the boundary layer are enclosed at all times. The salient large scale properties of the flow are:

- The existence of a strong transition (shock front) starting just before the nose of the ionopause interaction region (flat plate) and extending upwards as the flow transverses the region. Upstream from this transition the solar wind flow is not significantly affected by the ionospheric plasma.

- The existence of a viscous boundary layer over the flat plate. The velocity, density and temperature gradients established over the plate are carried downstream by the flow for several length scales beyond the flat plate.

- A stagnation zone preceding the leading edge of the flat plate where the reduction of the flow velocity results in an increased density and temperature.

These features are qualitatively similar for all choices of model parameters $\left(M_{\mathrm{o}}, R e\right)$. However, their extent and amplitude depends on the parameters $M_{\mathrm{o}}$ and $R e$. In the following subsection we will compare results for different values of the model parameters. 


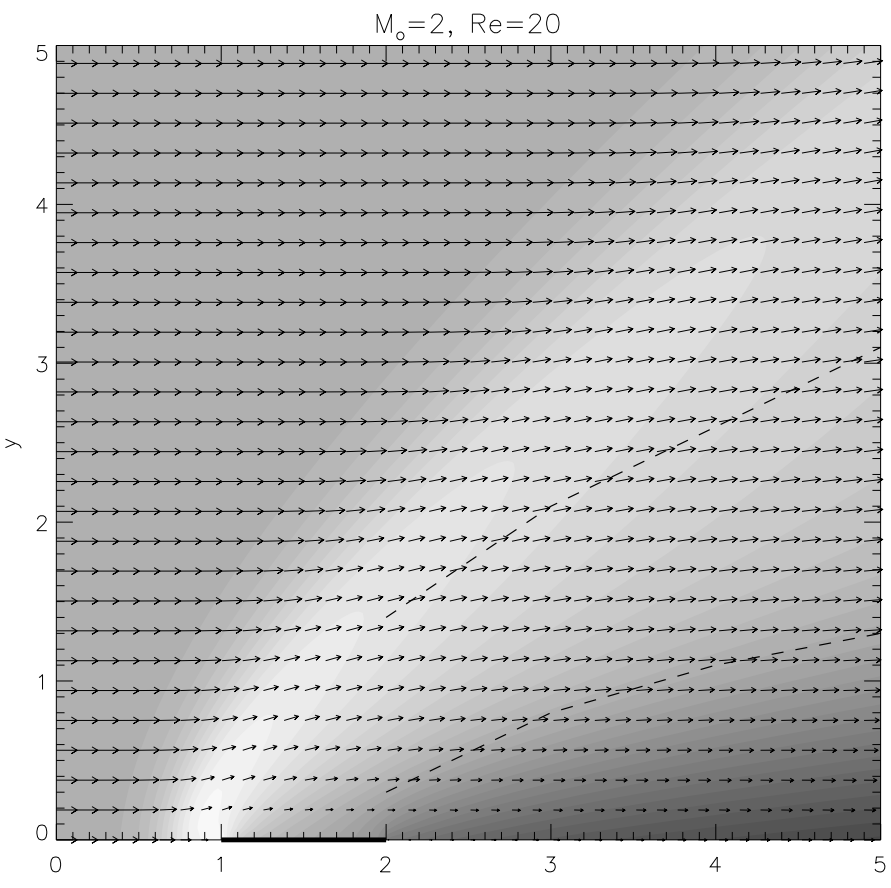

Fig. 2. Velocity field and density contours for the flow past a flat plate model for the ionopause for the fiducial case $M_{\mathrm{o}}=2$ and $R e=20$. The dark line between 1 and 2 in the bottom boundary indicates the location of the flat plate. The velocity field is shown by the black arrows and the shaded contours represent the gas density with lighter shaded regions having the highest density. The lower dashed line indicates the height of the boundary layer and the upper dashed line indicates the location of the bow shock as inferred from the measurements of Venera 10 (Romanov et al. 1979).

In Fig. 2, corresponding to our fiducial model, the location of the shock is indicated by the uppermost points at which the Solar wind velocity is noticeably changed from its freestream values. Owing to the strong viscous forces, $R e=20$, the shock is not an abrupt transition. This can be seen from the density contours in Fig. 2 which show the density increasing gradually by less than a factor of 2 , from its value in the freestreaming flow to its maximum value, indicated by the lightest shaded region in the figure.

The behavior of the flow properties over the computational domain can be seen in detail in the vertical profiles shown in Fig. 3 for our fiducial case, with $M_{\mathrm{o}}=2$ and $R e=20$. Profiles shown for $V, T$ and $\rho$ in the top, middle and bottom panels respectively, illustrate the behavior of the different properties with height above the flat plate; their actual value is not given by the numbers on the abscissa axis. All profiles are shifted and in some cases scaled to appear at their corresponding $x$ location and they start, at the top boundary, from the corresponding value for the free streaming flow; the value they reach at the bottom boundary depends on whether they are over the flat plate or not. For example, the $V_{x}$ profile starting from $x=2$ at the bottom boundary actually shows $V_{x} / 2+2$. The corresponding $T$ and $\rho$ profiles start from $x=2$ at the top boundary of the middle and bottom panel respectively, and show $T+1$ and $\rho+1$. The rest of the properties are constructed similarly.

As can be seen in Fig. 3, the vertical profile of the $x$ component of the velocity, $V_{x}$, indicates the existence of 2 transitions as one approaches the ionopause. The velocity first goes from its freestreaming value to a slightly lower one, about $25 \%$ less depending on the $x$-position. We identify this transition as the

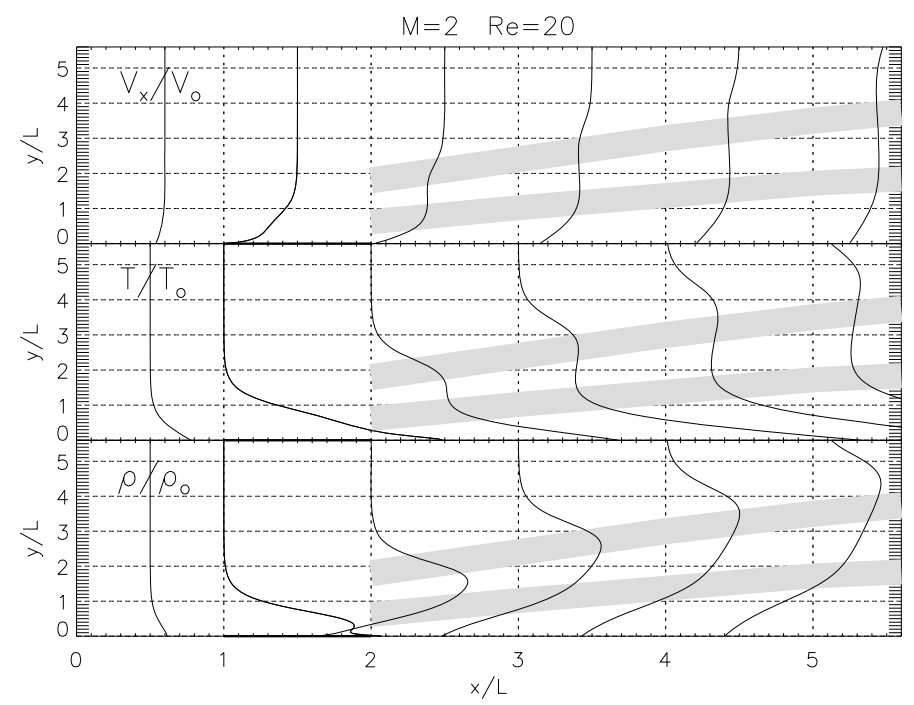

Fig. 3. Vertical profiles of flow properties at different positions along the plate for the fiducial case $M_{\mathrm{o}}=2$ and $R e=20$. The top panel shows the $x$ component of the velocity; temperature and density profiles are shown in the middle and bottom panels respectively. The dark line between 1 and 2 in the bottom boundary indicates the location of the flat plate. In all panels, the lower shaded region indicates the range of boundary layer thickness as inferred from the measurements of Venera 10 and Mariner 5. The corresponding range for the location of the bow shock is denoted by the upper shaded region. Profiles are intended to illustrate the behavior of the different properties with height above the flat plate and do not show their actual value directly (see text).

shock produced by the boundary layer as an obstacle. As one continues to approach the ionopause, the velocity remains more or less constant until, in the vicinity of the bottom boundary, it decreases in a viscous boundary layer. The velocity decreases to zero at the flat plate. Downstream from the flat plate, $V_{x}$ only decreases to a fraction of its value above the boundary layer, as there is no condition forcing it to zero at the bottom boundary. The boundary layer is advected downstream from the interaction region following the flow characteristics. Near the inner edge of the plate, where the solar wind flow first interacts directly with the ionospheric plasma, the shock front and the boundary layer are not clearly separated. Upstream from the interaction region and near the bottom boundary, there is a stagnation region as the flow slows down to near zero velocities over the flat plate. This can also be seen from Fig. 4, which shows the variation of flow properties along the $x$ coordinate at the very first gridpoint above the flat plate.

The corresponding changes in other flow properties are as follows. In temperature, shown in the middle panel of Fig. 3, the shock front is marked by a fast increase, by about $50 \%$ over its freestream value. A similar increase is also found for the gas density, as seen in the bottom panel of Fig. 3. In the boundary layer near the bottom boundary, viscous dissipation results in elevated temperatures with the gas heating to more than twice its freestream value. The gas in turn expands significantly as shown in the bottom panel of Fig. 3 reaching, at the bottom boundary, densities of about one half the value in the freestreaming flow. The behavior of density and temperature in the stagnation region and near the flat plate is also shown in Fig. 4, which shows the variation of flow properties along the $x$ coordinate just above the flat plate. Note in this figure that the effect of the interaction region are felt significantly upstream from its leading edge.

In the interest of brevity we have chosen not to show results for a case with a value of $M_{\mathrm{o}}$ smaller than our fiducial model. 


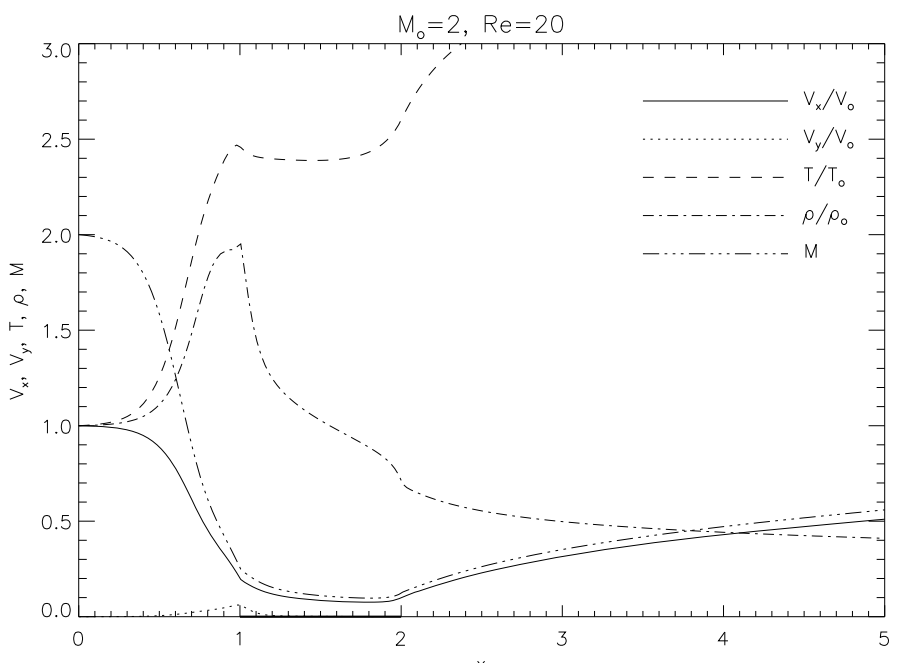

Fig. 4. Profiles of flow properties along the $x$ coordinate, at the first gridpoint above the plate, for the fiducial model with $M_{\mathrm{o}}=2$ and $R e=$ 20. The dark line between 1 and 2 in the bottom boundary indicates the location of the flat plate.

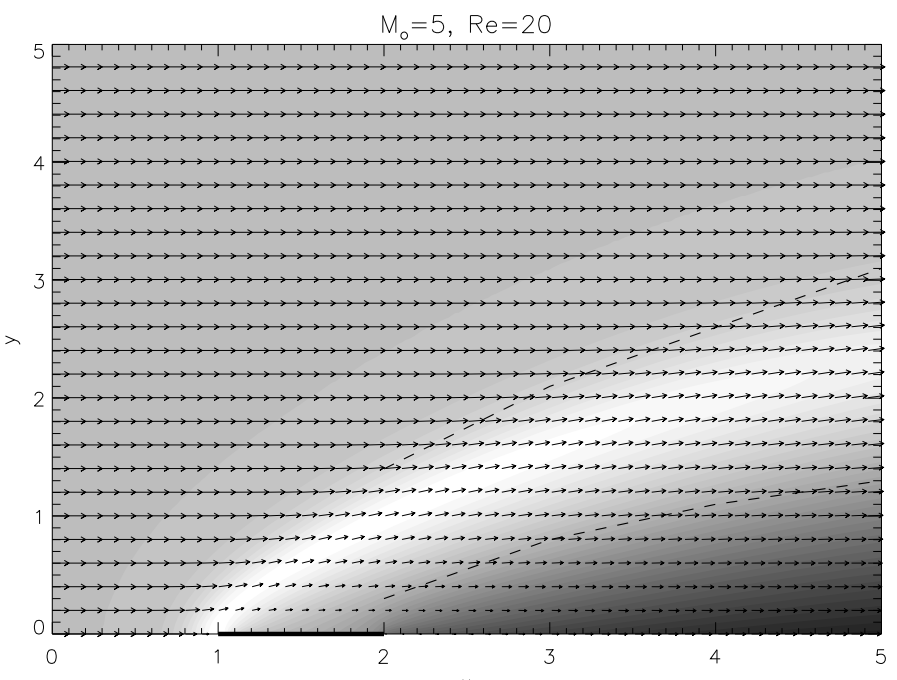

Fig. 5. Same as Fig. 2 but for a model having $M_{\mathrm{o}}=5$ and $R e=20$.

It cannot be much smaller than 2 , although a value of $M_{\mathrm{o}} \approx$ 1.5 could be argued for on the basis of the results of Spreiter \& Stahara (1980). It yields very similar results to our fiducial model. The only noticeable difference is a slightly higher shock front, with an asymptotic inclination approximately $10^{\circ}$ larger than that shown in Fig. 2.

\subsection{High $M_{\mathrm{o}}$ case}

To illustrate the effect of the Mach number of the incident flow, $M_{\mathrm{o}}$, on the flow properties, in Fig. 5 we show the steady state solution for the flow and in Fig. 6 we show the vertical profiles of $V_{x}, \rho$ and $T$ for a case having $M_{\mathrm{o}}=5$ and the same Reynolds number as our fiducial model, $R e=20$.

The main effect of increasing the incident Mach number is to modify the location of the shock front. As expected, a greater Mach number results in a shock front located closer to the obstacle, which is traced by the light shaded region in Fig. 5. Although the separation between the shock front and the top of the boundary layer is not as clear as in our fiducial case, the height of

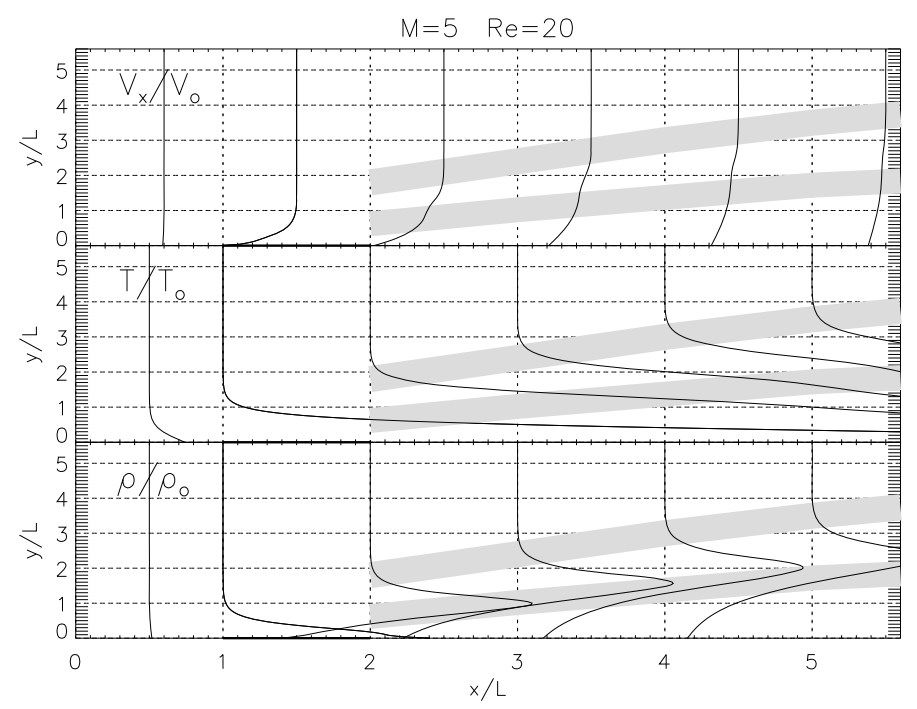

Fig. 6. Same as Fig. 3 but for a model having $M_{\mathrm{o}}=5$ and $R e=20$.

the boundary layer should increase. It is well known that with a faster freeflowing wind there is more kinetic energy to be dissipated in the boundary layer, leading to higher temperatures, and lower densities, near the bottom boundary. For hypersonic flow, the increase in temperature in the boundary layer leads to its expansion so that its height increases from the classical, subsonic, Blasius expression, which is inversely proportional to the square root of the Reynolds number, to:

$\delta \propto \frac{M_{\mathrm{o}}}{\sqrt{R e}}$

where the height of the boundary layer, $\delta$, is enhanced by the value of the Mach number (Anderson 1989). Also evident in Fig. 6 is the fact that the boundary layer disappears faster as one moves downstream from the flat plate. At a distance behind the magnetic poles 4 times the size of the interaction region, there is almost no velocity gradient associated with the boundary layer.

\subsection{High Re case}

If the Mach number is kept at $M_{\mathrm{o}}=2$, increasing the Reynolds number has the effect of reducing the viscous effects on the flow and the height of the boundary layer decreases in comparison to our fiducial model according to Blasius expression. This is seen in Figs. 7 and 8 which show the flow geometry and vertical profiles of $V_{x}, T$ and $\rho$, respectively, for a case having $R e=100$ and the same Mach number as our fiducial model, $M_{\mathrm{o}}=2$.

Reducing the extent of the boundary layer has the additional effect of making the obstacle appear less blunt to the incoming solar wind. The asymptotic opening angle of the shock front is more or less the same as in our fiducial model, however, the height of the shock front is significantly reduced to almost a half of its value in our fiducial model. A similar decrease in the extent of the stagnation region upstream from the inner edge of the flat plate is found.

The increased Reynolds number reduces the viscous spreading of the shock so that the jump in velocity and other flow properties across the shock front are more abrupt for the case with $R e=100$, shown in Fig. 8, than for our fiducial model. 


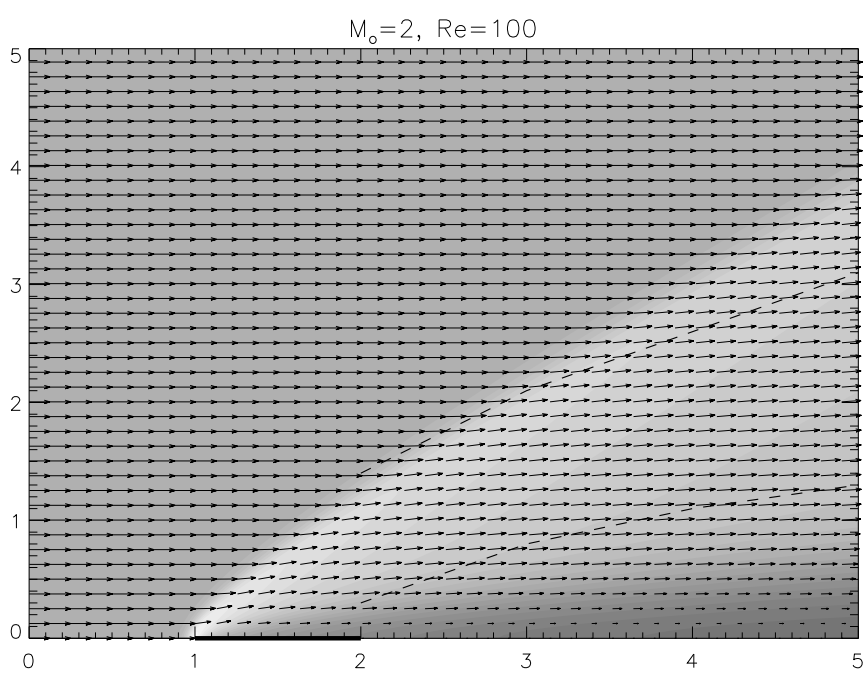

Fig. 7. Same as Fig. 2 but for a model having $M_{\mathrm{o}}=2$ and $R e=100$.

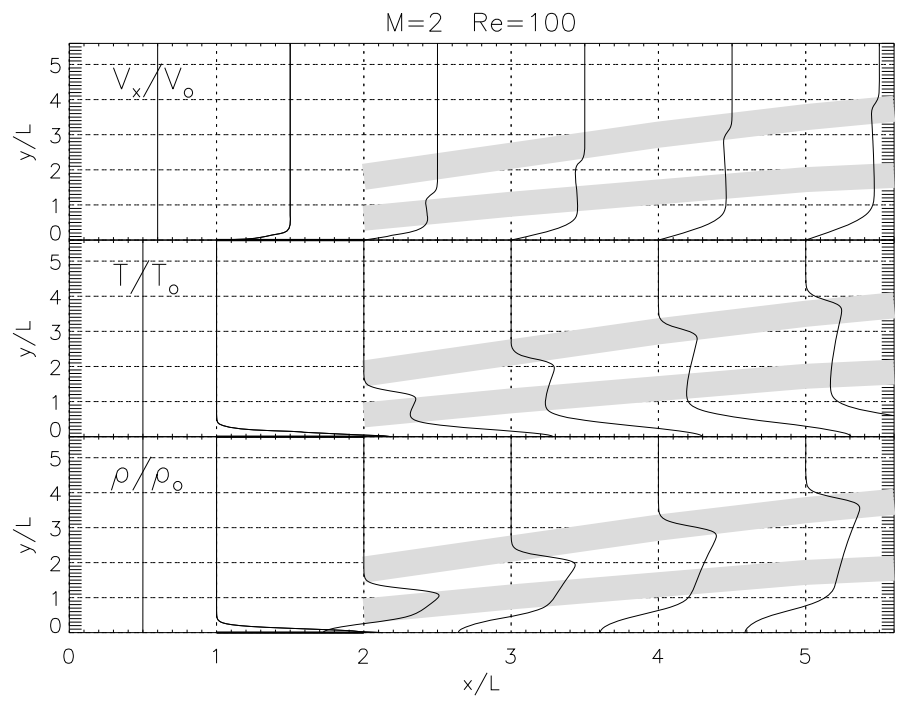

Fig. 8. Same as Fig. 3 but for a model having $M_{\mathrm{o}}=2$ and $R e=100$.

\subsection{Low Re case}

To illustrate the effect of reducing the Reynolds number in our fiducial model, effectively increasing viscous forces, in Figs. 9, 10 and 11 we show results from our simulations using $M_{\mathrm{o}}=2$ and $R e=5$.

Several features of the flow are significantly affected by the decrease in $R e$ in comparison to our fiducial model. The dispersion of the shock front over a greater extent, and the increased height of the boundary layer, results in no clear separation between these two transitions throughout the flow. The boundary layer height, $\delta$, increases roughly by a factor of 2 , according to Eq. (16). As seen in Fig. 10 the change in flow properties, particularly $T$ and $\rho$, associated with the shock front are now observed at greater heights.

The effectively increased viscosity also affects the extent of the stagnation zone preceding the inner edge of the flat plate, as can be seen in Fig. 11. To address this issue we have extended our computational domain upstream from the flat plate to $x_{\min }=-1$. And even so, judging from the fast decline of $V_{x}$ as soon as we leave the left boundary, it appears we have not caught the full extent of the stagnation zone. With the current version of

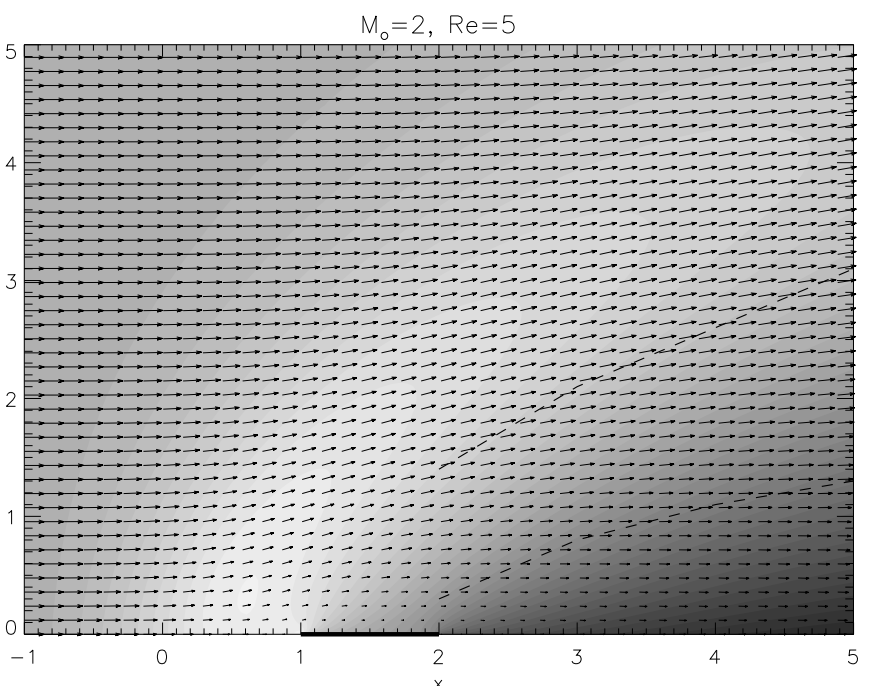

Fig. 9. Same as Fig. 2 but for a model having $M_{\mathrm{o}}=2$ and $R e=5$.

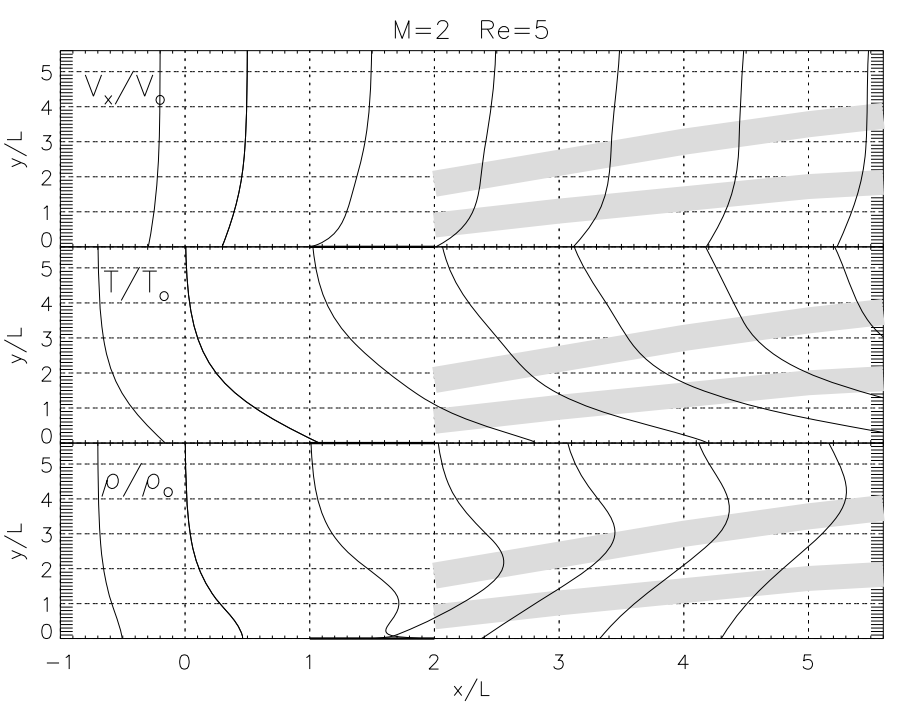

Fig. 10. Same as Fig. 3 but for a model having $M_{\mathrm{o}}=2$ and $R e=5$.

our computational code this is too demanding. Nevertheless the tendency is clear; viscosity gives rise to significant effects on the flow a considerable distance upstream from the region of direct interaction between the solar wind and Venus ionospheric plasma.

\section{Discussion}

Several features of our results can be compared to in situ measurements carried out during the fly-by of the Venera 10 (Romanov et al. 1979) and Mariner 5 (Bridge et al. 1967) spacecraft. Figure 12 shows the trajectory of Venera 10 and profiles of the measured ion temperature and speed. Figure 13 shows the trajectory of Mariner 5 and profiles of the measured ion thermal speed, ion number density and bulk velocity during the fly-by.

Both Venera 10 and Mariner 5 detected a transition in the plasma properties, located between the shock front and the ionopause, as the spacecraft approached the planet. Perez-de-Tejada (1986a) has labeled this as the intermediate transition, as shown in Figs. 12 and 13. It marks the beginning of a significant decrease in gas velocity and density as well as in increase in gas temperature. As argued by Perez-de-Tejada (1986a) 


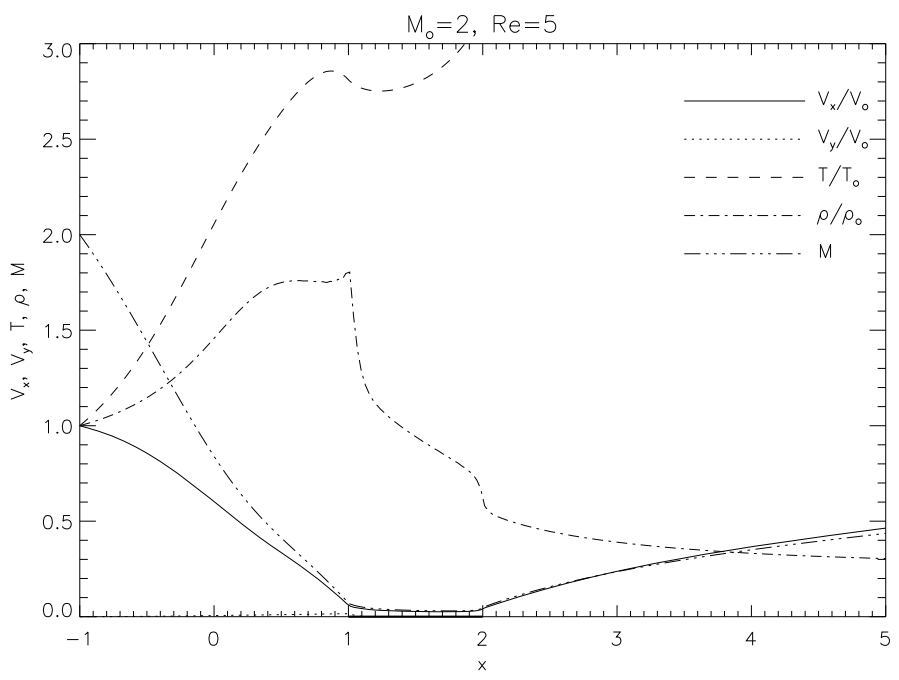

Fig. 11. Same as Fig. 4 but for a model having $M_{\mathrm{o}}=2$ and $R e=5$.
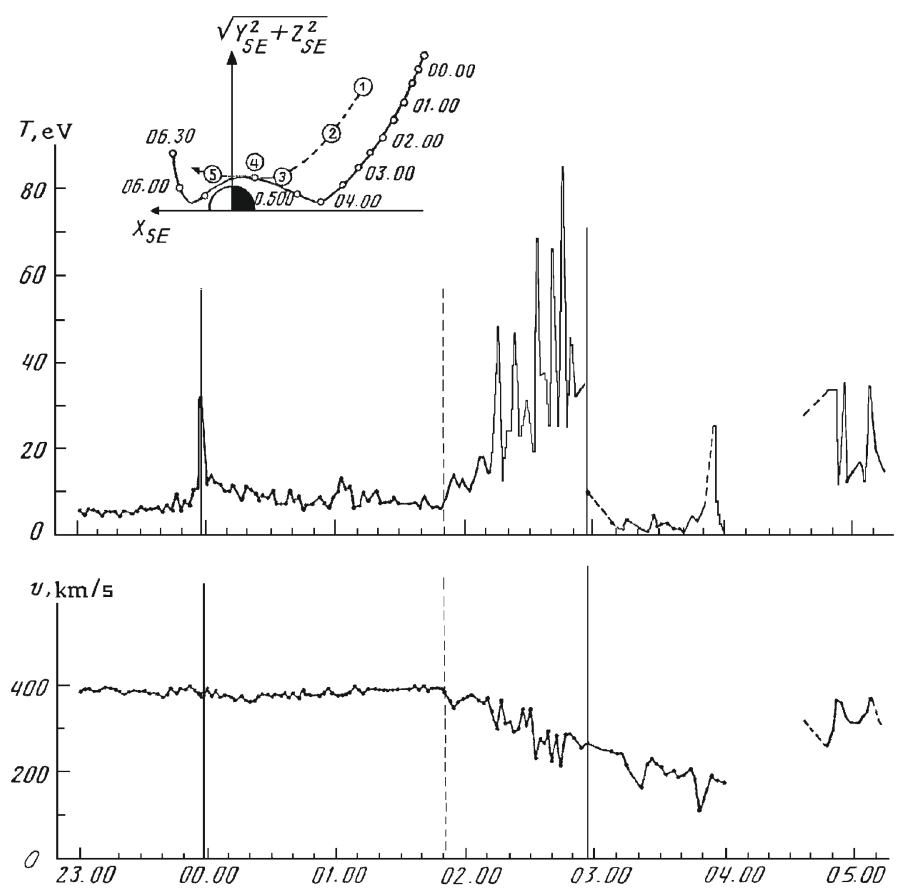

Fig. 12. Trajectory and measured plasma properties during the fly-by of Venera 10 (Romanov et al. 1979).

a simple explanation for this is the existence of a boundary layer over the planet's magnetic poles. In this model, the intermediate transition represents the height of the boundary layer.

We have superimposed in Figs. 3, 6, 8 and 10 a pair of shaded regions indicating a range in the position of the shock front and the height of the boundary layer as derived from the measurements of Venera 10 (Romanov et al. 1979) and Mariner 5 (Bridge et al. 1967). The range in each case is defined by the difference in the measurements of both spacecraft, with Mariner 5 having measured a slightly higher shock front and boundary layer. Defining the precise location of the shock front or the height of the boundary layer from the results of our numerical simulations is not straightforward; however, by comparing the velocity profiles in Figs. 3 and 8, it is clear that a choice of $R e=20$ gives a better agreement with the measured height of the boundary layer.

Comparing the boundary layer thickness and shock front height as derived from the measurements of Venera 10 and

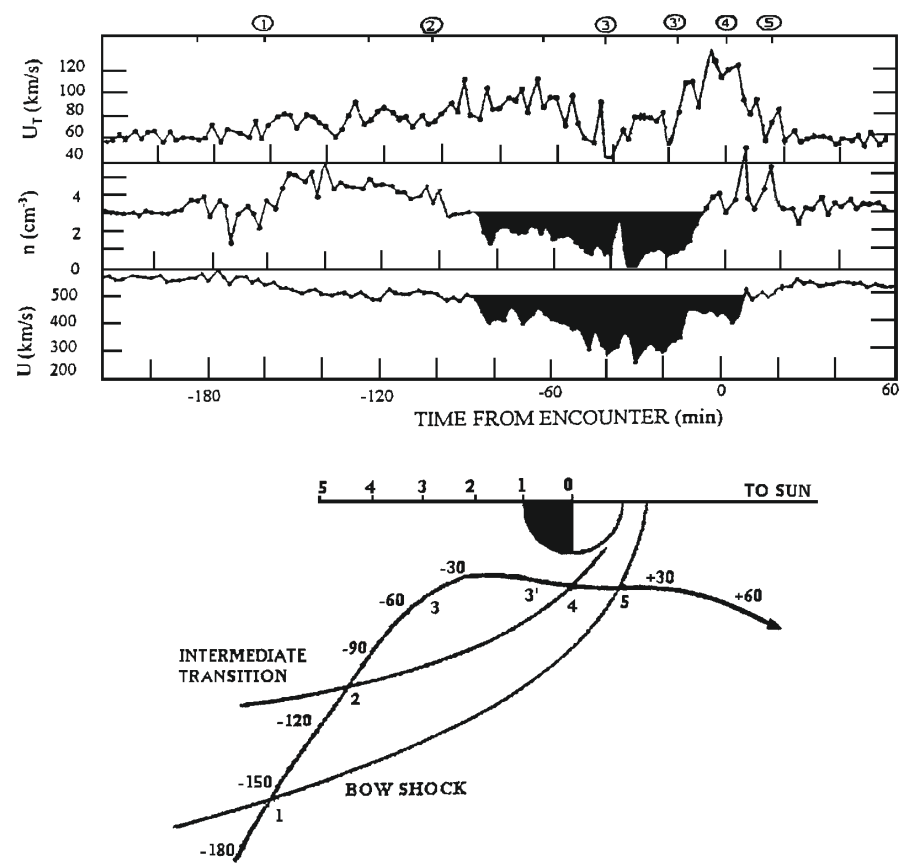

Fig. 13. Trajectory and measured plasma properties during the fly-by of Mariner 5 (Bridge et al. 1967).

Mariner 5 with the results from models with different Mach number (Figs. 3 and 6) it is clear that the observed position of the shock front cannot be accounted for if one assumes that the Mach number over the planet's magnetic poles is much greater than 2. A similar conclusion is also reached if one looks at the increase in temperature and the decrease in density from the top of the boundary layer to the bottom. Measurements by Venera 10 as reported by Romanov et al. (1979) indicate that the temperature increases approximately by a factor of 4 as one transverses the boundary layer behind the planet, as can be seen in Fig. 12. For the model with $M_{\mathrm{o}}=5$ and $R e=20$, Fig. 6 , the calculated temperature increase at those distances is much greater. According to Bridge et al. (1967) the densities measured by Mariner 5 in its pass by Venus decrease by a factor of 3 across the boundary layer, as seen in Fig. 13. Once again this is not consistent with the results shown in Fig. 6 for the case with $M_{\mathrm{o}}=5$ and $R e=20$.

In summary, by comparing the positions of the shock front and the intermediate transition measured by Venera 10 (Romanov et al. 1979) and Mariner 5 (Bridge et al. 1967) it appears that models characterized by a value of $M_{\mathrm{o}}$ near 2 and $R e$ of approximately 20 are in good agreement. Among the models we have computed, we have found that a model characterized by $M_{\mathrm{o}}=3$ and $R e=20$ gives the best agreement with the measured properties of the flow above and downstream of the magnetic polar regions of Venus. The vertical profiles of $V_{x}, T$ and $\rho$ for this "best fit" model are shown in Fig. 14.

\section{Conclusions}

We have carried out 2D numerical simulations of the hydrodynamical, viscous interaction between the solar wind and the ionosphere of Venus at the magnetic polar regions, modeling the ionopause as a flat plate. Our results indicate that as a consequence of this interaction one expects to encounter the following: a) a boundary layer of thickness $\delta$ given by Eq. (16) and increasing as one moves downstream. The gradients in velocity and other flow properties generated as the flow passes over the flat plate are advected far downstream past the magnetic poles 


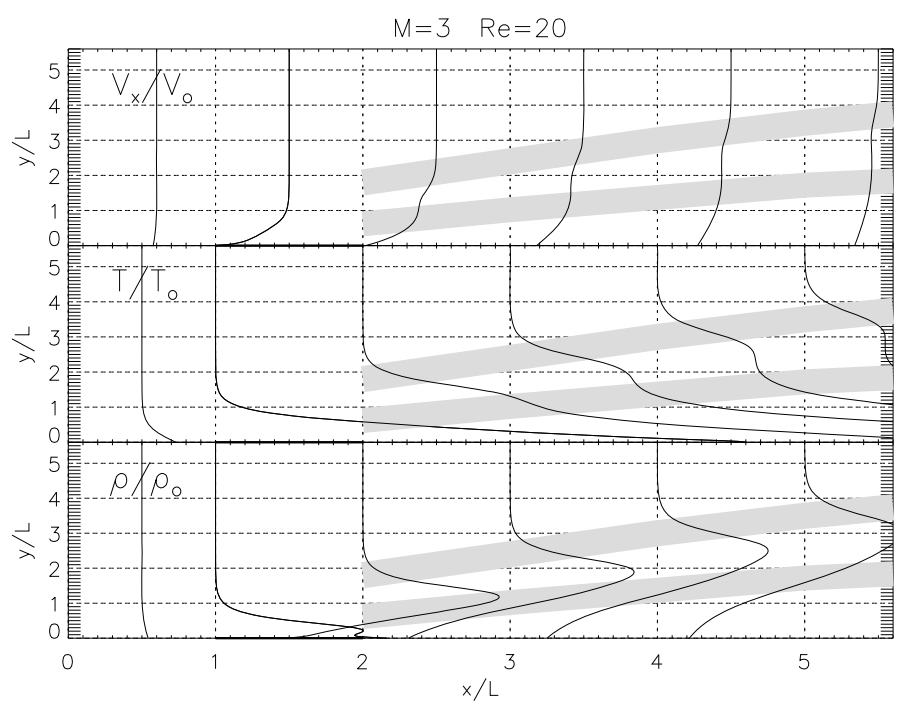

Fig. 14. Same as Fig. 3 but for a model with $M_{\mathrm{o}}=3$ and $R e=20$.

of the planet; b) a shock front located over the boundary layer at a location depending both on the Mach number of the incident flow as well as on its Reynolds number; and c) a stagnation zone located at low heights, upstream of the inner edge of the region of direct interaction between the solar wind and the ionospheric plasma, modeled in our study as a flat plate. The amplitude and extent of these features depends essentially on the incoming flow dimensionless numbers, $M_{\mathrm{o}}$ and $R e$.

By comparing our results for various choices of model parameters with the location of the shock front and boundary layer height as derived from the measurements of Venera 10 and Mariner 5, it is possible to rule out scenarios with a Reynolds numbers much greater than $R e=20$. This is illustrated in Fig. 15, where the height of the shock front, $H_{\text {sh }}$, and the thickness of the boundary layer, $\delta$, is plotted as a function of $R e$ for a series of models with different values of the incident Mach number, $M_{\mathrm{o}}$. The values plotted correspond to $H_{\mathrm{sh}}$ and $\delta$ evaluated at $x=5$. Also shown in Fig. 15 as shaded regions is the range of $H_{\mathrm{sh}}$ and $\delta$ as defined by the in situ measurements of Venera 10 (Romanov et al. 1979) and Mariner 5 (Bridge et al. 1967). It is evident that, independently of the precise value of the Mach number of a given model, a low value of the Reynolds number, around $R e \approx 20$, is necessary to fit the measured position of the shock front and the thickness of the boundary layer.

To our knowledge, this is the first attempt to include viscous forces in the numerical simulation of the solar wind-Venus interaction. As such, there are many pending issues still to be addressed that could have potentially important consequences on the details of the solutions obtained. Among these, some of the more important are the following: a) we have completely neglected the effect of the magnetic field on the flow; b) geometric effects due to the curvature of the ionosphere are not addressed; this is related to the choice of boundary conditions at the bottom boundary of our simulation box when not over the flat plate. This analysis will allow us to study, among other problems, the important issue of the variation in the shock front location with the solar cycle (Slavin et al. 1980); c) further analysis is required of the relation of the shock front found in our results and the shock

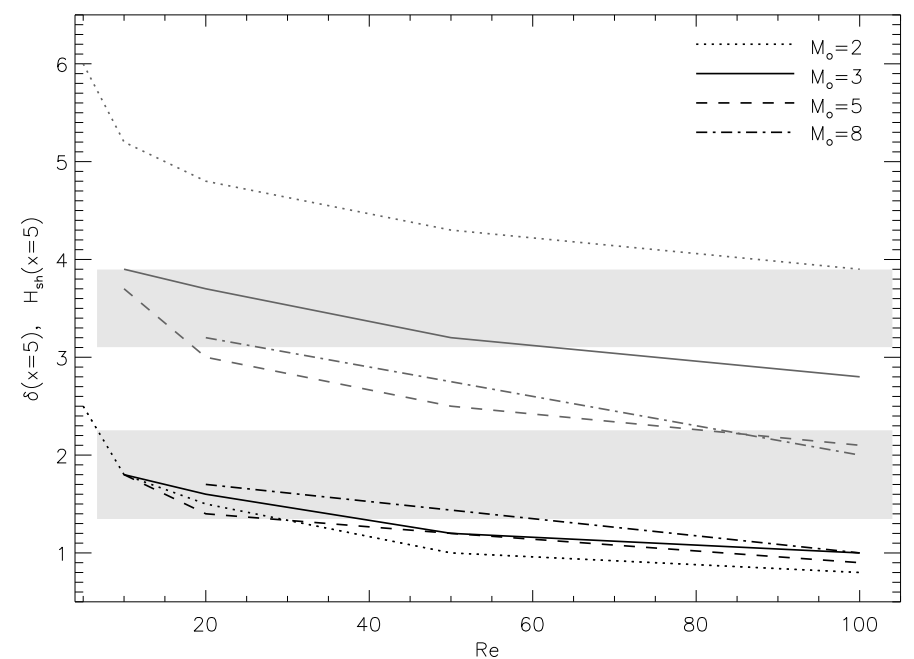

Fig. 15. Direct comparison of the height of the shock front (gray set of lines) and the thickness of the boundary layer (black set of lines) for different computed models, with the range for each quantity as derived from the measurements of Venera 10 and Mariner 5 spacecraft. All quantities correspond to values as $x=5$. The upper shaded region indicates the range of $H_{\mathrm{sh}}$ and the lower shaded region denotes the range of $\delta$.

front as measured by the Mariner 5 and Venera 10 spacecraft; and d) 3D effects and incoming flow time dependence need to be assessed. These issues will be addressed in future work.

Acknowledgements. MRR and EDM acknowledge support from DGAPAUNAM project INI05-505-3. The authors thank Dr. H. Aceves for useful criticism to early versions of the paper.

\section{References}

Anderson, J. D. 1989, Hypersonic and high temperature gas dynamics (New York: McGraw-Hill)

Anderson, J. D. 1995, Computational fluid dynamics: The basics with applications (New York: McGraw-Hill)

Brace, L. H., Theis, R., \& Curtis, S. 1967, J. Geophys. Res., 87, 199

Bridge, H., Lazarus, A., Snyder, C. et al. 1967, Science, 158, 1669

Díaz-Méndez, E. 2007, B.Sc. Thesis, Facultad de Ciencias, UABC, Ensenada, México.

Isachenko, V. P., Osipova, V. A., \& Sukomel, A. S. 1997, Heat Transfer (Moscow: Mir Publ.)

Miller, K., \& Whitten, R. 1991, Space Sci. Rev., 55, 165

Pérez-de-Tejada, H. 1986a, J. Geophys. Res., 91, 6765

Pérez-de-Tejada, H. 1986b, J. Geophys. Res., 91, 8039

Pérez-de-Tejada, H. 1995, Space Sci. Rev., 72, 655

Pérez-de-Tejada, H. 1999, ApJ, 525, L65

Pérez-de-Tejada, H. 2001, J. Geophys. Res., 106, 211

Pérez-de-Tejada, H. 2004, J. Geophys. Res., 109, A01106

Pérez-de-Tejada, H. 2006, J. Geophys. Res.,111, A11105

Quest, K. B., Shapiro, V. D., Szegö, K., \& Dobe, Z. 1997, Geophys. Res. Lett., 24, 301

Romanov, S. A., Smirnov, V., \& Vaisberg, O. 1979, Cosmic Res., 16, 603

Shapiro, V. D., Szegö, K., Ride, S., \& Shevchenko, V. 1995, J. Geophys. Res., 100,21289

Shefer, R., Lazarus, A., \& Bridge, H. 1979, J. Geophys. Res., 84, 2109

Slavin, J. A., Elphic, R. C., Russel, C. T., et al. 1980, J. Geophys. Res., 85, A7625

Spreiter, J., \& Stahara, S. 1980, J. Geophys. Res., 85, 7715

Taylor, H. A., \& Grebowsky, J. M. 1985, J. Geophys. Res., 90, 7415

Whitten, R., Mc Carmick, P. T., Merritt, D., et al. 1984, Icarus, 60, 317 